Ann. Biol. anim. Bioch. Biophys., I97r, 11 (3), 427-434.

\title{
GARACTÉRISTIQUES BIOCHIMIQUES DU RÉTICULUM SARCOPLASMIQUE DU MUSCLE DE PORC POST-MORTEM
}

\author{
J. CHARPENTIER ( $\left.{ }^{\mathbf{1}}\right)$ \\ Station de Recherches sur la Viande, \\ Centre de Recherches de Clermont-Ferrand, I. N. R. A., \\ 63 - Saint-Genès-Champanelle \\ RÉSUMÉ
}

Cette expérience avait pour but, d'une part d'étudier le captage du $\mathrm{Ca}^{++}$par les microsomes du réticulum sarcoplasmique ainsi que leur activité $\mathrm{ATPasique} \mathrm{à} \mathrm{différents} \mathrm{temps} \mathrm{post} \mathrm{mortem}$ et d'autre part de préciser l'influence du $\mathrm{pH}$ et de la température sur la dénaturation de ces microsomes. Les résultats obtenus montrent que, post mortem, le réticulum sarcoplasmique perd plus ou moins rapidement son aptitude à accumuler le calcium en fonction de la vitesse de diminution du pH. L'étude de l'influence du $\mathrm{pH}$ et de la température montre que l'abaissement du pH provoque une dénaturation du réticulum qui est accentuée lorsque la température est relativement élevée. Les ions calcium sont donc relargués d'autant plus rapidement que la chute de $\mathrm{pH}$ est plus brutale et qu'elle se produit à une température proche de celle de l'animal vivant. Un relargage important des ions $\mathrm{Ca}$ consécutif à une évolution anormale du pH musculaire provoque l'activation post mortem de la myosine ATPase et est donc à l'origine de l'activité ATPasique intense et de l'apparition très rapide de la rigor mortis dans les muscles de porc exsudatif.

Depuis la mise en évidence d'un facteur relaxant dans le muscle, par Marsh en I952, de très nombreux travaux ont permis de montrer que ce facteur s'identifiait aux vésicules du réticulum sarcoplasmique. Il est bien établi en effet, qu'en présence d'ATP, les triades du réticulum peuvent accumuler les ions calcium et en abaisser ainsi la concentration en dessous du niveau critique nécessaire pour la contraction (EBashi, I96I ; Hasserbach et Marinose, I96I ; WeBber et al., I963). C'est ainsi que des fibres glycérinées, des myofibrilles ou de l'actomyosine peuvent se contracter ou se relaxer en fonction de la concentration en ions calcium du milieu (EBASHI, I96I ; WEBER, I959; WEBER et HERZ, I96I ; BENDALI, I963). Si le mode d'action du réticulum vis-à-vis du calcium est actuellement bien élucidé dans les conditions physiologiques nos connaissances quant à son activité post mortem sont, par contre,

(1) Les demandes de tirés à part doivent être adressées à la Station de Recherches sur la Viande, I. N. R. A., 63-Saint-Genès-Champanelle, M. CHARPENTIER étant décédé le 30 avril I97I. 
très limitées. En effet, jusqu'alors seuls GREASER et al. (I967) ont montré qu'après la mort, les fractions lourdes du réticulum sarcoplasmique du muscle de porc perdent progressivement leur aptitude à accumuler le calcium. La vitesse plus ou moins grande du relargage des ions calcium pourrait vraisemblablement expliquer les différences importantes que l'on enregistre dans la cinétique d'installation de la rigor mortis dans cette espèce. Dans le cas des viandes exsudatives notamment la diminution à la fois du taux d'ATP et du $\mathrm{pH}$ sont particulièrement rapides. Le $\mathrm{pH}$ atteint alors sa valeur ultime très peu de temps post mortem alors que la température du muscle est voisine de celle de l'animal vivant. Ainsi est-il concevable de supposer que la conjonction d'un $\mathrm{pH}$ bas et d'une température relativement élevée soit susceptible de dénaturer les structures membranaires particulièrement fragiles du réticulum et de provoquer ainsi un relargage du calcium. Le but de notre travail est précisément de vérifier la validité de cette hypothèse.

\section{MATÉRIEL ET MÉTHODES}

Dans un premier temps nous avons étudié, à différents temps post mortem l'activité ATPasique des microsomes et leur aptitude à accumuler le calcium. Les microsomes sont isolés sur des échantillons de 30 grammes du muscle Longissimus Dorsi de porcs Large White pesant entre Ioo et I Io kgs. Ces échantillons sont prélevés au niveau de la $4^{\mathrm{e}}$ et $6^{\mathrm{e}}$ vertèbre dorsale, à $0, \mathrm{r}, 2$, 3 et $\mathbf{2 4}$ heures post mortem. Après détermination du $\mathrm{pH}$, on procède immédiatement à l'extraction des microsomes.

Dans un deuxième temps nous avons étudié d'une part l'activité ATPasique des microsomes en fonction du $\mathrm{pH}$ et d'autre part l'influence de la conjonction $\mathrm{pH}$-température sur l'activité ATPasique des microsomes et leur aptitude à accumuler le calcium. Pour cela nous avons extrait des microsomes du muscle Long du Cou prélevé immédiatement après l'abattage de l'animal. Le choix de ce muscle est motivé par la valeur systématiquement élevée de son pH initial (ce qui n'est pas le cas du Longissimus Dorsi). Dès son obtention, la suspension de microsomes est congelée dans l'azote liquide et conservée à $-20^{\circ} \mathrm{C}$. Des essais préalables nous avaient montré que dans ces conditions l'activité des microsomes est parfaitement conservée pendant une durée d'un mois.

\section{Extraction des microsomes}

Vingt grammes environ de tissu musculaire sont broyés au moyen d'un homogénéiseur Biorex pendant deux minutes en présence de 30 volumes d'un milieu saccharose 0,25 M tamponné par de l'histidine $0,005 \mathrm{M}$ à $\mathrm{pH}_{7,0}$. Les myofibrilles sont séparées par centrifugation pendant 20 minutes à $\mathrm{I} 000 \mathrm{~g}$. Le surnageant est centrifugé pendant $3^{\circ}$ minutes à $8000 \mathrm{~g}$ ce qui permet d'isoler les mitochondries. Le surnageant ainsi obtenu est centrifugé pendant I heure à $30000 \mathrm{~g}$. Le culot de centrifugation constitue la fraction correspondant aux microsomes " lourds ". Toutes ces centrifugations sont effectuées à $0^{\circ} \mathrm{C}$ au moyen d'une centrifugeuse MSE HS I8. L'examen microscopique (microscope optique) et la détermination de l'activité cytochrome oxydase montrent que la fraction microsomale ainsi obtenue est peu contaminée par des mitochondries légères. Le culot de centrifugation est mis en suspension dans le milieu saccharose-histidine de façon à obtenir une concentration en protéines (déterminée par la méthode du biuret) de $4 \mathrm{mg} / \mathrm{ml}$. Cette suspension est immédiatement congelée dans l'azote liquide et conservée à $-20^{\circ} \mathrm{C}$.

\section{Fixation du calcium par les microsomes et activité ATPasique}

L'incubation des microsomes est effectuée à $37^{\circ} \mathrm{C}$ dans le milieu suivant : o, I $\mathrm{M} \mathrm{KCl,} 5 \mathrm{mM}$ oxalate de potassium, $5 \mathrm{mM} \mathrm{ATP}, 0,05 \mathrm{mM}\left(\mathrm{CaCl}_{2}+{ }^{45} \mathrm{CaCl}_{2}\right)$ tamponné par $20 \mathrm{mM}$ d'histidine à $\mathrm{pH} 7,4$. Pour étudier l'influence du $\mathrm{pH}$ sur l'activité ATPasique et la fixation du ${ }^{45} \mathrm{Ca}, \mathrm{l}^{1}$ 'histidine est remplacée par un tampon tris-maléate $50 \mathrm{mM}$ dont la composition permet de faire varier le pH du milieu d'incubation entre 5,5 et 7,4. La quantité de ${ }^{45} \mathrm{CaCl}_{2}$ (fourni par le Commissariat 
à 1'Énergie atomique, activité spécifique $210,8 \mathrm{mCi} / \mathrm{g}$ ) est calculée de telle façon que l'activité de la solution mesurée au compteur Tricarb Packard soit de l'ordre de $50000 \mathrm{cpm} / \mathrm{ml}$. La réaction est déclenchée par addition des microsomes de telle sorte que la concentration finale en protéine du milieu d'incubation soit de $0,2 \mathrm{mg} / \mathrm{ml}$. Toutes les trois minutes, pendant 15 minutes, $2 \mathrm{ml} \mathrm{de}$ ce milieu sont filtrés sous vide à travers des filtres Millipore $\mathrm{HA}_{45}$. Simultanément $0,5 \mathrm{ml}$ d'acide trichloracétique Io p. Ioo sont ajoutés à $2 \mathrm{ml}$ de ce même milieu. Le phosphore minéral est dosé dans le surnageant obtenu par centrifugation, par la méthode de Fiske et Subarow.

La filtration sur filtre Millipore permet de séparer les microsomes du milieu d'incubation. Les ions calcium fixés aux microsomes restent sur le filtre alors que les ions calcium libres passent dans le filtrat. Les radioactivités du filtre et du filtrat sont mesurées au moyen d'un compteur à scintillation liquide Tricarb Packard.

\section{RÉSULTATS ET DISCUSSION}

\section{I. - Fixation du calcium par les microsomes \\ et activité ATPasique des microsomes à différents temps post mortem}

Il est bien établi que dans le cas du muscle de porc la vitesse de diminution du pH post mortem présente une très grande variabilité. Dans le cas des viandes exsudatives notamment la chute de $\mathrm{pH}$ est particulièrement rapide. Aussi nous a-t-il semblé intéressant d'étudier l'évolution de la liaison du calcium aux microsomes en fonction de la rapidité de chute du $\mathrm{pH}$ post mortem. La figure I montre les variations du taux de calcium lié dans le cas de muscles à chute de $\mathrm{pH}$ rapide et de muscles à chute de $\mathrm{pH}$ lente. Il apparaît nettement que le taux de Ca lié đépend étroitement de la valeur du $\mathrm{pH}$. Immédiatement après l'abattage de l'animal lorsque le $\mathrm{pH}$ est
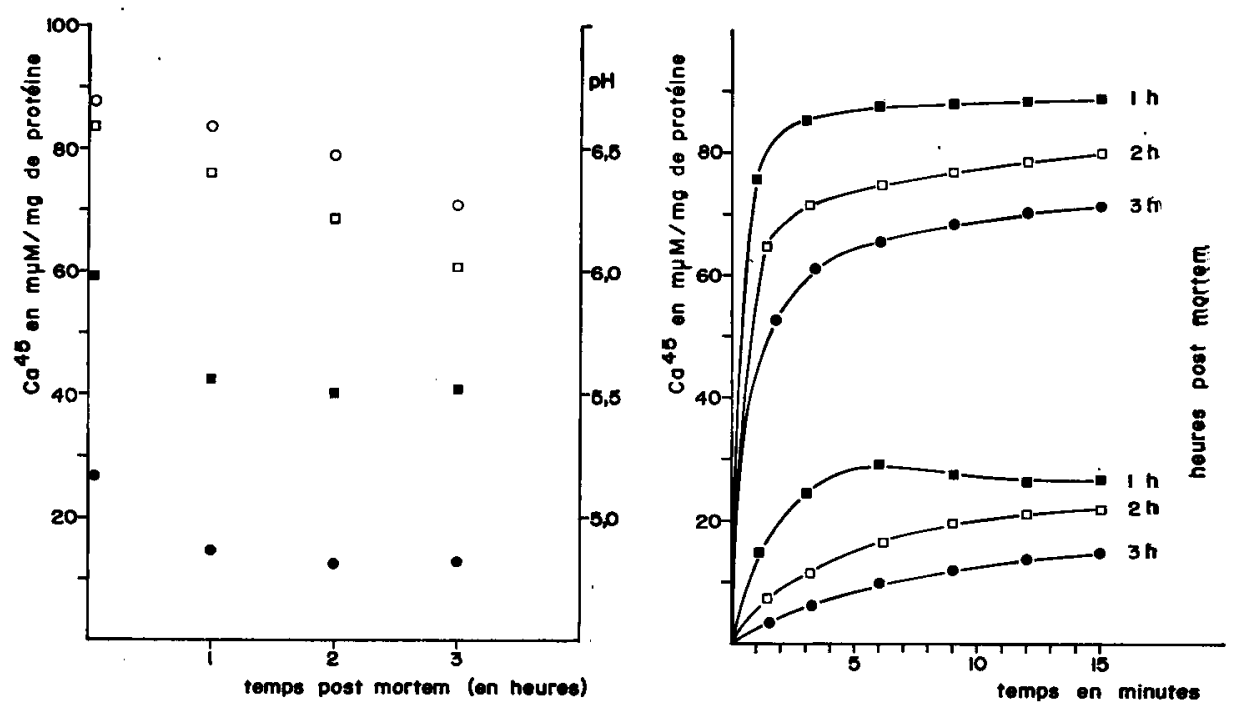

Frg. I. - Evolution post mortem $d u p H$ musculaire et $d u{ }^{45} \mathrm{Ca}$ lié aux microsomes chute de $\mathrm{pH}$ lente $:{ }_{45}^{\mathrm{pH}} \mathrm{Ca}$
chute de $\mathrm{pH}$ rapide $:{ }_{{ }^{45} \mathrm{Ca}}^{\mathrm{CH}}$

FIG. 2. - Cinétique de fixation $d u{ }^{45} \mathrm{Ca}$ par les microsomes à différents temps post mortem 
élevé, c'est à dire en 1'occurrence égal à 6,6 , la quantité de calcium lié est de $88 \mathrm{~m} \mu \mathrm{M}$ par mg de protéine. Par contre, lorsque le $\mathrm{pH}$ initial n'est que de 6,0 , le calcium lié ne représente que $27 \mathrm{~m} \mu \mathrm{M}$ par $\mathrm{mg}$ de protéine.

Dans le cas où le $\mathrm{pH}$ diminue lentement le taux de Ca lié demeure élevé puisque pour un $\mathrm{pH}$ de 6,o, 3 heures post mortem, $70 \mathrm{~m} \mu \mathrm{M}$ de Ca par mg de protéine sont encore retenues par les microsomes. Lorsque le $\mathrm{pH}$ chute rapidement le taux de $\mathrm{Ca}$ lié par contre s'effondre rapidement puisque pour un $\mathrm{pH}$ de 5,6, I heure post mortem il n'est que de I4 muM par mg de protéine. La comparaison des quantités de $\mathrm{Ca}$ accumulées par les microsomes pour les valeurs voisines du $\mathrm{pH}$ musculaire, montre que les différences ainsi observées ne sont pas imputables à l'influence du $\mathrm{pH}$ mais correspondent très vraisemblablement à l'action conjuguée du $\mathrm{pH}$ et de la température ainsi qu'à des activités intrinsèques variables des microsomes selon les types musculaires.

La figure 2 montre nettement que lorsque le taux de Ca lié diminue, la vitesse de fixation du $\mathrm{Ca}$ par les microsomes est également plus faible.

La mise en évidence d'une perte progressive, et plus ou moins rapide, de l'aptitude des microsomes à accumuler le calcium corrobore pleinement les résultats de GREASER et al. (I967). Il convient, à ce propos, de remarquer toutefois que les quantités de Ca fixées par les microsomes sont nettement inférieures à celles citées par ces auteurs qui ont utilisé une concentration en $\mathrm{Ca}$ de $0,2 \mathrm{mM}$ alors que dans nos conditions expérimentales celle-ci n'est que de I5 $\mu \mathrm{M}$. Cette concentration présente a notre avis l'intérêt de mieux correspondre aux conditions physiologiques. En effet, selon WEBER et al. (Ig66) la capacité maximum de fixation du $\mathrm{Ca}$, sans oxalate, est de I20 à I5o $\mathrm{m} \mu \mathrm{M}$ par $\mathrm{mg}$ de protéine. Or, en utilisant des concentrations en $\mathrm{Ca}$ dans le milieu d'incubation de ${ }_{5} 5 \mu \mathrm{M}$, nous obtenons précisément des taux de Ca lié qui restent inférieurs à la capacité de saturation en l'absence d'oxalate.

Pour des raisons analogues, des concentrations du même ordre ont d'ailleurs déjà été utilisées par plusieurs auteurs (Scales et McInTosh, I968) (HaRigaya et al., I968).

Compte tenu de la diminution de l'aptitude des microsomes à accumuler les ions $\mathrm{Ca}$ il en résulte post mortem un relargage de ces ions qui permet l'activation de l'ATPase myofibrillaire et le déclenchement de la rigor mortis. A ce titre le pourcentage élevé de $\mathrm{Ca}$ libre dans les muscles à bas $\mathrm{pH}$ post mortem rapide rend parfaitement compte de la rapidité d'installation de la rigor mortis dans les viandes exsudatives. Comme l'indique le tableau I la diminution de 1'activité ATPasique est beaucoup plus importante dans le cas des muscles à chute de pH rapide. Il convient de remarquer toutefois que l'activité A'TPasique déterminée en présence d'oxalate ne présente que peu d'analogie avec l'activité qui se manifeste au niveau des microsomes in situ. En effet, les microsomes sont, semble-t-il le siège de deux activités ATPasiques distinctes (INESI et WATANABE, I967) à savoir une activité de base indépendante du taux de calcium, et une activité correspondant au mécanisme d'accumulation du calcium. L'oxalate, en maintenant une concentration d'ions $\mathrm{Ca}$ libres constants à l'intérieur des microsomes permet d'éviter un relargage d'ions $\mathrm{Ca}$ et en conséquence une réaccumulation quasi simultanée dispendieuse d'énergie d'où une action apparemment inhibitrice de l'oxalate envers l'activité ATPasique des microsomes. 
TABLEAU I

Activité ATPasique des microsomes à différents temps temps post mortem

\begin{tabular}{|c|c|c|c|c|}
\hline \multirow{2}{*}{$\begin{array}{l}\text { Temps } \\
\text { post mortem } \\
\text { en heures }\end{array}$} & \multicolumn{2}{|c|}{$\begin{array}{l}\text { Muscle à chute de } \mathrm{pH} \\
\text { lente }\end{array}$} & \multicolumn{2}{|c|}{$\begin{array}{l}\text { Muscle à chute de } \mathrm{pH} \\
\text { rapide }\end{array}$} \\
\hline & $\begin{array}{c}\text { Activité } \\
\text { ATPasique } \\
\mu \mathrm{gP} / \text { protéine } / \mathrm{mn}\end{array}$ & $\mathrm{pH}$ & $\begin{array}{c}\text { Activité } \\
\text { ATPasique } \\
\mu \mathrm{gP} / \text { protéine/mn }\end{array}$ & $\mathrm{pH}$ \\
\hline 0 & 1,62 & 6,60 & 1,85 & 6,00 \\
\hline 1 & 1,38 & 6,40 & 0,60 & 5,55 \\
\hline 2 & 1,15 & 6,20 & 0,42 & 5,50 \\
\hline 3 & 1,10 & 6,00 & 0,40 & 5,50 \\
\hline
\end{tabular}

2. - Infuence du pH sur l'aptitude des microsomes à accumuler le calcium et sur leur activité ATPasique

L'évolution du taux de calcium lié en fonction du $\mathrm{pH}$ est indiqué par la figure 3 . Il apparaît nettement que la liaison du $\mathrm{Ca}$ aux microsomes diminue très rapidement lorsque le $\mathrm{pH}$ atteint des valeurs inférieures à 6,4 , pour devenir pratiquement nulle pour un $\mathrm{pH}$ de 5,5 . Ces résultats étayent donc l'hypothèse d'un relargage du Ca consécutif à l'abaissement du $\mathrm{pH}$ lors de la glycogénolyse.
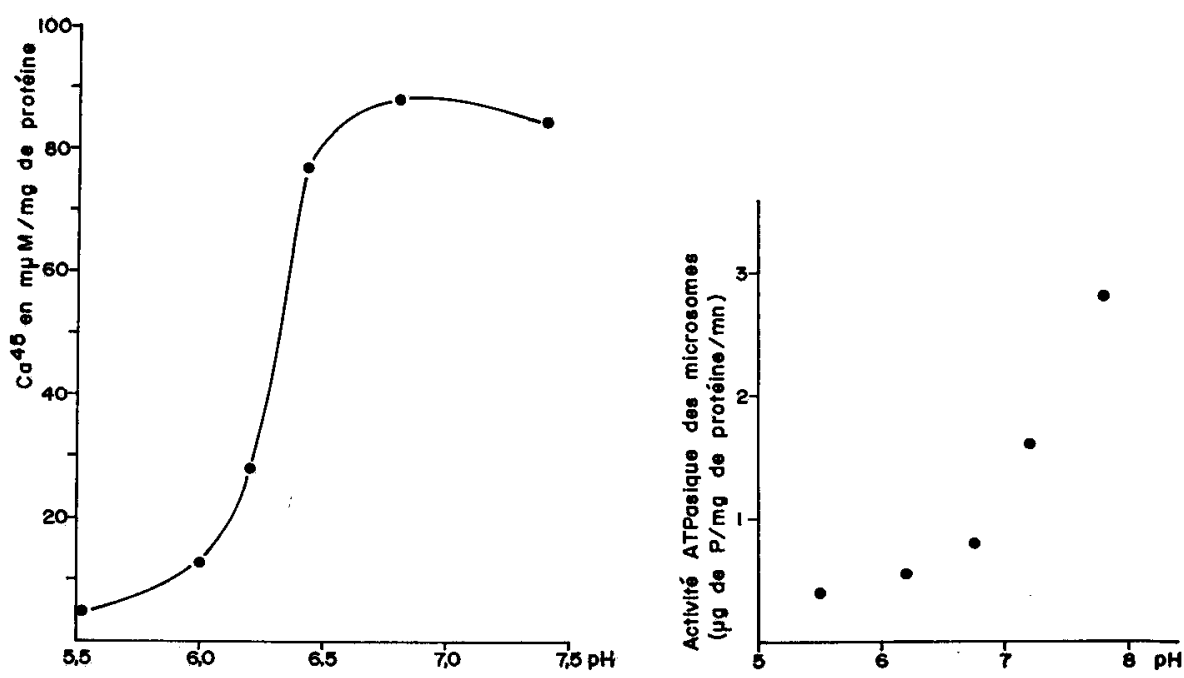

FIG. 3. - Influence $d u$ pH sur la fixation $d u^{45} \mathrm{Ca}$ par les microsomes

Fic. 4. - Activité ATPasique des microsomes en fonction du $p H$

L'étude de l'influence du $\mathrm{pH}$ sur l'activité ATPasique des microsomes (fig. 4) montre que celle-ci diminue très rapidement"entre $\mathrm{pH} 7,6$ et $\mathrm{pH} 6,8$ puis plus progressivement jusqu'à $\mathrm{pH} 5,5$. 
Il ne semble pas qu'il y ait une influence semblable du pH sur l'activité ATPasique des microsomes et sur leur aptitude à accumuler les ions Ca. En effet l'abaissement du pH entraîne une diminution de l'activité ATPasique avant que le taux de Ca lié ne soit affecté. Les modalités de l'action du $\mathrm{pH}$ sur l'activité des microsomes devront être élucidées au cours d'expériences ultérieures, en dissociant notamment 1'activité ATPasique de base de celle qui est liée effectivement au transport actif du calcium.

\section{3. - Action conjuguée du $p H$ et de la température sur la fixation du calcium par les microsomes et sur leur activité ATPasique}

Les microsomes étaient incubés à des $\mathrm{pH}$ de $5,4,6,0,6,5$ et 7,3 et à des températures de $20^{\circ} \mathrm{C}$ et $40^{\circ} \mathrm{C}$ pendant 30 à 60 minutes. Après incubation l'aptitude des microsomes à accumuler le calcium était déterminée dans les conditions optimales c'est-à-dire à $37^{\circ} \mathrm{C}$ et à $\mathrm{pH} 7,4$. Les résultats figurant au tableau 2 montrent nettement que la conjonction d'un bas $\mathrm{pH}$ et d'une température élevée diminue considérablement l'aptitude des microsomes à accumuler les ions Ca. Le maintien d'une température élevée contribue à accentuer l'influence néfaste de l'abaissement du $\mathrm{pH}$ sur la liaison des ions $\mathrm{Ca}$ au réticulum sarcoplasmique. L'action prolongée d'une température relativement élevée ralentit en effet la vitesse de captage des ions Ca par les microsomes. Compte tenu du fait qu'après incubation les déterminations de l'aptitude des microsomes à accumuler le calcium étaient effectuées dans les conditions optimales de $\mathrm{pH}$ et de température, il apparaît, surtout dans le cas des essais réalisés à $+20^{\circ} \mathrm{C}$, que 1'influence inhibitrice de la diminution du $\mathrm{pH}$, est irréversible. L'abaissement du $\mathrm{pH}$ provoquerait donc une dénaturation du réticulum qui serait, semble-t-il, accentuée par une élévation de température.

TABLEAU 2

Calcium fixé (en mumoles par $\mathrm{mg}$ de protéine) après incubation des microsomes pendant 30 et 60 minutes dans différentes conditions de pH et de température

\begin{tabular}{|c|c|c|c|c|c|c|c|c|}
\hline \multirow{3}{*}{$\begin{array}{l}\text { Température } \\
\text { d'incubation }\end{array}$} & \multicolumn{8}{|c|}{$\mathrm{pH}$} \\
\hline & \multicolumn{2}{|c|}{5,4} & \multicolumn{2}{|c|}{6,0} & \multicolumn{2}{|c|}{6,5} & \multicolumn{2}{|c|}{7,3} \\
\hline & $30 \mathrm{mI}$ & $60 \mathrm{mn}$ & $30 \mathrm{mn}$ & $60 \mathrm{mn}$ & $30 \mathrm{mn}$ & $60 \mathrm{~mm}$ & $30 \mathrm{mn}$ & $60 \mathrm{mn}$ \\
\hline $20^{\circ} \mathrm{C}$ & 7 & 5 & 70 & 68 & 80 & 81 & 88 & 91 \\
\hline $40^{\circ} \mathrm{C}$ & 3 & 2 & 32 & 27 & 56 & 53 & 77 & 73 \\
\hline
\end{tabular}

En conclusion, il apparaît nettement que lors de l'évolution post mortem du muscle de porc, le réticulum sarcoplasmique perd plus ou moins rapidement son aptitude à accumuler le calcium, en fonction de la vitesse de diminution du $\mathrm{pH}$. L'étude de l'influence du $\mathrm{pH}$ et de la température montre que l'abaissement du $\mathrm{pH}$ provoque une dénaturation du réticulum qui est accentuée lorsque la température est relativement élevée. Les ions calcium sont donc relargués d'autant plus rapidement que 
la chute de $\mathrm{pH}$ est plus brutale et qu'elle se produit à une température proche de celle de l'animal vivant. Un relargage important des ions Ca consécutif à une évolution anormale du $\mathrm{pH}$ musculaire provoque 1'activation post mortem de la myosine ATPase et est donc à 1'origine de l'activité ATPasique intense et de l'apparition très rapide de la rigor mortis dans les muscles de porc exsudatif.

Reçu pour publication en mars 1971.

\section{SUMMARY}

BIOCHEMICAL, CHARACTERISTICS OF THE SARCOPLASMATIC RETICULUM OF THE PIG MUSCLE POST MORTEM

The current investigation was undertaken in order to study the $\mathrm{Ca}^{++}$uptake by the microsomes of the sarcoplasmic reticulum, the microsomal ATPase activity at various times post mortem, and the effect of $\mathrm{pH}$ and temperature on microsomal denaturation.

The microsomes were isolated from sections of the longisssimus dorsi pig muscle at hrs $0, \mathrm{I}, 2,3,24$ post-mortem. Microsomal Ca-binding activity was estimated using radio-calcium $\mathrm{Ca}^{45}$. After filtration on a Millipore filter, estimation of the filtre and filtrate radioactivities enabled to determine the amount of $\mathrm{Ca}^{++}$taken up by the microsomes.

The microsomal Ca biding ability was found to strongly depend on the speed of $\mathrm{pH}$ decrease post mortem.

With slowly declining $\mathrm{pH}$ values, the amount of bound $\mathrm{Ca}$ remains high (70 $\mathrm{m} \mu \mathrm{M}$ per $\mathrm{mg}$ protein for $\mathrm{pH} 6.0$ at $\mathrm{hr} .3$ post mortem). With sharply decresing $\mathrm{pH}$ values, the amount of bound $\mathrm{Ca}$ is markedly depressed ( $14 \mathrm{m \mu M}$ par mg protein for $\mathrm{pH} 5.6$ at hr. I).

Our results also point out the severe decrease in bound $\mathrm{Ca}$ occuring for $\mathrm{pH}$ values lower than 6.4 (reaching o for $\mathrm{pH}$ 5.5). Moreover, when low $\mathrm{pH}$ and high temperature conditions meet, the capacity of microsomal $\mathrm{Ca}$ binding suffers a marked decrease. $\mathrm{Ca}^{++}$ions therefore are unbound al the faster as the $\mathrm{pH}$ decrease is the sharper and occurs at temperatures approching that of the live animal.

The considerable unbinding of $\mathrm{Ca}^{++}$ions following abnormal $\mathrm{pH}$ changes induces the post mortem activation of ATPase myosine, and therefore appears as the factor responsible for the high ATPase activity and rapid occurrence of the rigor mortis in exsudative pig muscles.

\section{RÉFÉRENCES BIBLIOGRAPHIQUES}

Bendall J. R., 1963. Physiology and chemistry of muscle. A. R. C. low temperature. Res. Stat. Memoirs202.

Eвashi S., r96r. Calcium binding activity of vesicular relaxing factor. J. Biochem., 50, 236-244.

Greaser M. L., Cassens R. G., Hoekstra W. G., 1967. Changes in oxalate simulated calcium accumulation in particulate fractions from post mortem muscle. J.A gr. Food Chem., 15, I II2-I I 7 .

Harigaya S., Ogawa Y., Sugita H., I968. Calcium binding activity of microsomal fraction to rabbit red muscle. J. Biochem., 63, 324-331.

Hasselbach W., Makinose M., ig6r. Die Calciumpumpe der " Erschlaffungsgrana " des Muskels und ihre Abhängigkeit von der ATP-spaltung. Biochem. $Z$., 333, 518-528.

InEsI G., Watanabe S., I967. Temperature dependance of ATP hydrolysis and calcium uptake by fragmented sarcoplasmic membranes. Arch. Biochem. Biophys., 121, 665-671.

MarSh B. B., I952. The effects of adenosine triphosphate on the fibre volume of a muscle homogenate. Biochim. Biophys. Acta., 9, 247-260.

Scales B., McIntosh D. A. D., 1968. Studies on the radiocalcium uptake and the adenosine triphosphatases of skeletal and cardiac sarcoplasmic reticulum. J. Pharmac. Exp. Therap., 160, 249-260.

WeBER A., r959. On the role of calcium in the activity of adenosine-5' triphosphate hydrolysis by actomyosine. J. Biol. Chem., 234, 2764-2769. 
WEBER A, HERz $R$, 1965. Requirement for ealcium in the symaeresis of myofibrils. Biochem. Biophys, Res. Comm, 6, 364.

WEBER A., HERz R, Reiss I., I963. On the mechanism of the relaxing effect of fragmented sarcoplasmic reticulum. J. Gen. Phystol, 46, 679 .

WEBER A, HERZ R., REISS I, 1966 . Study of the kinetics of calcium transport by isolated fragmented sarcoplasmic reticulum."Blochem, $Z$, 345, 329-369. 